\title{
Duality and Global Symmetries
}

\author{
Fernando Quevedo ${ }^{\text {a* }}$ \\ ${ }^{a}$ Instituto de Física, \\ Universidad Autónoma de México \\ Apartado Postal 20-364, 01000 \\ México D.F., México \\ Email: fernando@ft.ifisicacu.unam.mx
}

\begin{abstract}
Duality symmetries are reviewed. A sufficient condition for duality is the existence of a global symmetry. This can be used as a guideline to systematically prove duality between different field theories. Bosonization and $T$ - duality, for abelian and non-abelian global symmetries, are discussed in detail as well as duality for general antisymmetric tensor field theories. In this case, the presence of topological defects break the global symmetry but duality survives manifesting itself in a different way. Open questions and current limitations of this approach to prove all known dualities are discussed.
\end{abstract}

To the hundreds of thousands of Guatemalan citizens who sacrificed their lives during more than three decades of civil war, especially to those I had the privilege to know and love, and who I now miss.

\section{INTRODUCTION}

The current excitement about duality in field and string theories leads us to believe that duality is indeed an exact symmetry of many physical systems. But in many cases we can only rely on indirect evidence for duality rather than proving it explicitly. We would like to know precisely when two theories can actually be shown to be dual to each other. The general answer to this question is unknown but a sufficient condition for the existence of duality is the existence of a global symmetry in the original theory. Given any field theory with a global symmetry, there is a well defined prescription to find the dual theory. This brings us a long way towards explicitly showing the equivalence between two different theories and puts many dualities on an equal setting. In particular we will see that bosonization can be proven exactly in the same way as we prove $T$ duality.

First I would like to remark that, before the recent developments, duality had a long history. It

\footnotetext{
*Lectures given at 33rd Karpacz Winter School. Fepruary
} 1997. Preprint IFUNAM FT97-07, hep-th/9706210 was known to Maxwell that his equations in vacuum were invariant under the exchange of electric and magnetic fields, but it was until 1931 that Dirac used this duality to introduce the magnetic monopole in order to keep electric/magnetic duality valid in the presence of sources. Independent of this, Kramers and Wannier discovered temperature duality in the 2D Ising model allowing them to learn about the phase structure of the model before Onsager's solution [1]. This was then generalized to several lattice and continous models leading to the Higgs/confinement duality of 't Hooft and Mandelstam as well as the MontonenOlive conjecture for duality in $4 \mathrm{D}$ gauge theories over two decades ago [2]. At about the same time, independent to this development, Luther and Preschel realized that fermions and bosons in $2 \mathrm{D}$ systems are equivalent [3]. Bosonization has lead to many applications in condensed matter physics and high energy theory. All these 'dualities' had in common that they relate strong to weak coupling (or temperature) and that they exchange elementary states by topological defects or solitons (electric/magnetic charges) although they were discovered independently and using dif- 
ferent techniques.

During the past decade, Witten introduced non-abelian bosonization [1], hidden symmetries of supergravity theories were discovered containing duality [5] and, in string theory, the subject started with the realization of $R \leftrightarrow 1 / R$ duality in toroidal compactifications [6], [7]. Later on, this duality was understood in terms of the standard duality of 2D non-linear sigma models studied in supergravity [8], [9] and it was extended to more general backgrounds, including backgrounds with non-abelian isometries [10]. Duality originating from symmetries of the string 2D sigma model is presently known as $T$-duality. These developments lead also to the conjecture of a possible duality in condensed matter systems such as the quantum Hall effects, almost ten years ago [11. This has acquired more relevance recently after the spectacular experimental evidence found last year [12].

The subject of this school has its origin on the more recent developments related to strong/weak coupling or $S$-duality in supersymmetric field and string theories which started during the present decade and are the main subject of research at present 13], [14. Most of the recent developments will be reviewed by the other speakers. I will concentrate on the concrete question: under which circumstances can we actually prove duality between two different theories?. In string theory the methods presented here apply mostly to $T$-duality. For $S$-duality we cannot prove it explicitly because at present there is no nonperturbative formulation of string theory, that is why we have to rely on indirect evidence for this duality. However, one of these evidences is the duality between the two effective field theories of the massless modes of both (dual) strings. That corresponds to duality of antisymmetric tensor field theories which we will discuss in these lectures.

The aim of these lectures is then to review the explicit prescriptions for dualization. If there is any field theory with a global symmetry then we can dualize it by gauging the corresponding symmetry and introducing a Lagrange multiplier constraint that sets the gauge field strength to zero [9]. Depending on the nature of the symmetry we can have abelian or non-abelian duality and depending on the nature of the original degrees of freedom we can have duality for scalar fields in 2D (T-duality), for electromagnetism or arbitrary antisymmetric tensors ( $S$-duality), or duality for fermions i.e. bosonization.

The lectures are organized as follows. In section 2 we discuss abelian duality for two specific systems: arbitrary antisymmetric tensors in any dimension and fermions in two and higher dimensions. The particular case of rank zero tensors in $2 \mathrm{D}$ is discussed in some detail ( $T$-duality) and examples, such as toroidal compactifications and $2 \mathrm{D}$ black holes, are presented. In section 3 duality is extended to the nonabelian case. Here we restrict ourselves to non-abelian $T$-duality and nonabelian bosonization. Some proposed generalizations of non-abelian duality are also presented. Finally in section 3 we discuss duality for the systems of section 1 when the presence of topological defects, such as magnetic monopoles, break the original global symmetry. For pedagogical reasons, we illustrate the general case by studying mostly QED. First we explictly show duality in the presence of magnetic monopoles. Then, after these monopoles condense, we present the effective Lagrangian describing the so-called 'confining string' description of the confining phase of the theory, we also prove that duality is manifest even in this case. We close the section with the examples of $T$-duality after vortex condensation for the bosonic string case and describe how duality is not broken by the Kosterlitz-Thouless phase transition. We close the lectures mentioning some open questions for the techniques presented here and some speculations.

\section{ABELIAN DUALITY}

We will review in this section duality in systems with abelian global symmetries. First we discuss the 2D case of the string worldsheet action. Then we generalize it to higher dimensions and fermionic systems.

\subsection{Abelian $T$-Duality}

We will briefly review here the standard duality corresponding to string backgrounds with abelian isometries. The worldsheet action for the bosonic 
string is:

$$
\begin{aligned}
S= & \frac{1}{4 \pi \alpha^{\prime}} \int \mathrm{d}^{2} z\left(Q_{M N}\left(X_{Q}\right) \partial X^{M} \bar{\partial} X^{N}\right. \\
& \left.+\frac{\alpha^{\prime}}{2} R^{(2)} \Phi\left(X_{Q}\right)\right)
\end{aligned}
$$

where $Q_{M N} \equiv G_{M N}+B_{M N} . G_{M N}$ and $B_{M N}$ being the metric and antisymmetric tensor of the target space theory and $\Phi$ is the dilaton field. They are 'couplings' in the 2D theory. $X^{M}$ are scalar fields of the 2D theory which are the coordinates in target space. $R^{(2)}$ is the scalar curvature in $2 \mathrm{D}$. We take the target space to have the critical dimension, $D=26$ for purely bosonic strings and $D=10$ for supersymmetric strings, in that case the action above would represent only (part of) the bosonic sector of the theory. In a background with $N$ commuting isometries, this can be written as

$$
\begin{aligned}
S= & \frac{1}{4 \pi \alpha^{\prime}} \int \mathrm{d}^{2} z\left(Q_{\mu \nu}\left(X_{\alpha}\right) \partial X^{\mu} \bar{\partial} X^{\nu}\right. \\
& +Q_{\mu n}\left(X_{\alpha}\right) \partial X^{\mu} \bar{\partial} X^{n}+Q_{n \mu}\left(X_{\alpha}\right) \partial X^{n} \bar{\partial} X^{\mu} \\
& \left.+Q_{m n}\left(X_{\alpha}\right) \partial X^{m} \bar{\partial} X^{n}+\frac{\alpha^{\prime}}{2} R^{(2)} \Phi\left(X_{\alpha}\right)\right) .
\end{aligned}
$$

Lower case latin indices $m, n$ label the isometry directions. Since the action depends on the $X^{m}$ variables only through their derivatives (there exists the abelian global symmetry $X^{m} \leftrightarrow X^{m}+$ constant), we can write it in first order form by introducing variables $A^{m}$ and adding an extra term to the action $\Lambda_{m}\left(\partial \bar{A}^{m}-\bar{\partial} A^{m}\right)$ which imposes the constraint $A^{m}=\partial X^{m}$. Integrating over the Lagrange multipliers $\Lambda_{m}$ returns us to the original action (1). On the other hand performing partial integration and solving for $A^{m}$ and $\bar{A}^{m}$, we find the dual action $\tilde{S}$ which has an identical form to $S$ but with the dual background given by [8], [15]

$$
\begin{aligned}
\tilde{Q}_{m n} & =\left(Q^{-1}\right)_{m n} \\
\tilde{Q}_{\mu \nu} & =Q_{\mu \nu}-Q_{\mu m}\left(Q^{-1}\right)^{m n} Q_{n \nu} \\
\tilde{Q}_{n \mu} & =\left(Q^{-1}\right)_{n}^{m} Q_{m \mu} \\
\tilde{Q}_{\mu n} & =-Q_{\mu m}\left(Q^{-1}\right)_{n}^{m} .
\end{aligned}
$$

To preserve conformal invariance, it can be seen that the dilaton field has to transform as $\tilde{\Phi}=\Phi-$ $\log \operatorname{det} G_{m n}$. Equations (2) reduce to the usual duality transformations for the toroidal compactifications in the case $Q_{m \mu}=Q_{\mu m}=0$ and can map a space with no torsion $\left(Q_{m \mu}=Q_{\mu m}\right)$ to a space with torsion $\left(\tilde{Q}_{m \mu}=-\tilde{Q}_{\mu m}\right)$.

Duality then states that the two dual theories defined by the actions $S[Q, \Phi]$ and $\tilde{S}[\tilde{Q}, \tilde{\Phi}]$ give the same partition function $Z$ and therefore they are physically equivalent. A more precise statement could be made by coupling the system to external sources so that the partition function and its derivatives with respect to the source are equivalent, meaning that also all the amplitudes are equivalent after a duality transformation.

An equivalent interpretation of the duality process just described is given by gauging the symmetry, replacing $\partial X^{m}$ with $D X^{m}=\partial X^{m}+A^{m}$ and the term $\int d^{2} z \Lambda_{m}\left(\partial \bar{A}^{m}-\bar{\partial} A^{m}\right)$ is added to the action. This extra term imposes the vanishing of the field strength $F$ of the gauge fields after integration over the Lagrange multipliers $\Lambda_{m}$. This implies that locally the gauge field must be pure gauge, $A^{m}=\partial X^{m}$. The gauge fixing can be done either by choosing the gauge fields to vanish or by taking $X^{m}=0$ (a unitary gauge). In both cases this reproduces the original action. The dual theory is obtained by instead integrating out the gauge fields and then fixing the gauge.

This is the prescription that can be systematically generalized: whenever there is a global symmetry we can gauge it and impose the corresponding field strength to zero. Changing the order of integrations we end up either with the original theory or with the dual theory where the Lagrange multiplier becomes the fundamental dual variable.

As an illustrative case we will discuss the circle compactifiction in more detail. In that case there is one isometry given by the shift on the circular coordinate. The corresponding metric component is just $R^{2}$, the radius of the circle. Therefore the duality equations above reduce to $R \leftrightarrow \alpha^{\prime} / R$ duality. Furthermore, being this a free 2D field theory, canonical quantization is straightforward, giving rise to a mass formula:

$$
\alpha^{\prime} M^{2}=n^{2} \frac{\alpha^{\prime}}{R^{2}}+m^{2} \frac{R^{2}}{\alpha^{\prime}}+2\left(N_{L}+N_{R}-2\right)
$$


The physical states are labeled by the integers $m, n$ where $P=n / R$ are the quatized momenta in the compact direction whereas $W=m R$ are the solitonic winding states with $m$ the number of times the string wraps around the circle. We can immediately observe that the mass formula is invariant under the duality transformation $R \leftrightarrow \alpha^{\prime} / R$ as long as the roles of the winding and momentum states are also exchanged. This is an example of a generic feature about duality that exchanges elementary with solitonic states.

This relation can be made more explicit by absorbing the radius $R$ in the definition of the coordinate $X$ which then has periodicity $X \equiv$ $X+2 \pi R$. In this situation we denote the dual coordinate by $\tilde{X}$. Probing the theory with external sources $J, \bar{J}$ the original action is

$S=\frac{1}{\pi \alpha^{\prime}} \int d^{2} z(\partial X \bar{\partial} X+J \bar{\partial} X+\bar{J} \partial X+\cdots)$

whereas the dual action is

$\tilde{S}=\frac{1}{\pi \alpha^{\prime}} \int d^{2} z(\partial \tilde{X} \bar{\partial} \tilde{X}-J \bar{\partial} \tilde{X}+\bar{J} \partial \tilde{X}+J \bar{J})(6)$

Notice the difference in the couplings to the currents and the presence of the contact term $J \bar{J}$ in the dual action ?. By comparing the coefficients of the currents we can see the relations $\partial X \leftrightarrow \partial \tilde{X}$ and $\bar{\partial} X \leftrightarrow-\bar{\partial} \tilde{X}$. This can be deduced by using the field equations for both $A, \bar{A}$ and $\Lambda \equiv \tilde{X}$ in the first order action and only means that (using 2D cartesian coordinates rather than complex ones) the two dual variables are related by the standard Poincaré duality of forms, $\partial_{\alpha} \tilde{X}=\varepsilon_{\alpha \beta} \partial^{\beta} X$. Which in turn implies that the field equations $\partial_{\alpha} \partial^{\alpha} X=0$ transform into Bianchi identities $\varepsilon_{\alpha \beta} \partial^{\alpha} \partial^{\beta} \tilde{X}$ and viceversa.

This information, combined with the wave equation $\partial \bar{\partial} X=\partial \bar{\partial} \tilde{X}=0$ implies that $X(z, \bar{z})=$ $X_{L}(z)+X_{R}(\bar{z})$ and $\tilde{X}(z, \bar{z})=X_{L}(z)-X_{R}(\bar{z})$. Comparing the zero-modes in the oscillator expansion of both variables:

$$
\begin{aligned}
X & =P \tau+W \sigma+\cdots \\
& =\frac{1}{2} p_{L} \ln z+\frac{1}{2} p_{R} \ln \bar{z}+\cdots
\end{aligned}
$$

${ }^{2}$ This term is precisely what is needed in order that both dual theories agree after taking care of time ordering in the corresponding amplitudes (I thank C.P. Burgess for explaining this to me.) where $p_{L, R} \equiv P \pm W$ are the left and right-moving momenta and the ellipsis refer to the oscillator terms. The dual variable is then

$\tilde{X}=\frac{1}{2} p_{L} \ln z-\frac{1}{2} p_{R} \ln \bar{z}+\cdots$

From this we can see that the two dual theories are related by the exchange $P \leftrightarrow W$, also that the hamiltonian (and so the mass formula) depends only on the combination $p_{L}^{2}+p_{R}^{2}$ which is duality invariant. Since the periodicity of $X$ gave rise to the quantization of the momenta $P$ and the momenta of the dual variable are given by $W$ we can then easily extract the periodicity of $\tilde{X}$ to be $\tilde{X} \equiv \tilde{X}+2 \pi \alpha^{\prime} / R$ (an overall integer factor $p$ can be considered, coinciding with the Dirac quantum [16], but modular invariance of the string theory restricts to $p=1$ ).

The simplest generalization of this system is compactification on a 2D torus instead of a 1D circle. This extension is straightforward but very interesting. Duality gives the transformation of the 2D matrix $G_{i j}+B_{i j} \leftrightarrow\left(G_{i j}+B_{i j}\right)^{-1}$. But now, since the purely antisymmetric tensor part of the action (1) is topological, there is also a symmetry $B \rightarrow B+$ integer, where $B$ is defined from $B_{i j}=B \varepsilon_{i j}$. Since this symmetry does not commute with duality, they together generate an infinite dimensional discrete symmetry. This is better expressed in terms of the complex parameters or moduli:

$$
\begin{aligned}
T & =B+i \sqrt{G} \\
U & =\frac{G_{12}}{G_{22}}+i \frac{\sqrt{G}}{G_{22}}
\end{aligned}
$$

$U$ is the standard modular parameter of any geometrical 2D torus and it is usually identified as the 'complex structure' modulus. $T$ is the 'Kähler structure' modulus (since the torus is a complex Kähler manifold) and its imaginary part measures the overall size of the torus, since $G$ is the determinant of the $2 \mathrm{D}$ metric. It then plays the same role as the radius $R$ for the $1 \mathrm{D}$ circle.

In terms of $T$ and $U$ we can write the left- and right-moving momenta as:

$p_{L}^{2}=\frac{1}{2 U_{2} T_{2}}\left\|\left(n_{1}-n_{2} U\right)-T\left(m_{2}+m_{1} U\right)\right\|^{2}$ 
$p_{R}^{2}=\frac{1}{2 U_{2} T_{2}}\left\|\left(n_{1}-n_{2} U\right)-\bar{T}\left(m_{2}+m_{1} U\right)\right\|^{2}$

The mass formula, depending on $p_{L}^{2}+p_{R}^{2}$, shows the following symmetries:

$U \rightarrow \frac{a U+b}{c U+d} \quad T \rightarrow \frac{a T+b}{c T+d} \quad T \leftrightarrow U$.

Where $a, b, c, d$ are integers satisfying $a d-b c=$ 1. The first transformation is the standard $S L(2, \mathbb{Z})_{U}$ 'modular' symmetry of $2 \mathrm{D}$ tori and is independent of string theory; it is purely geometric. The second transformation is a stringy $S L(2, \mathbb{Z})_{T}$ named $T$-duality and it is a generalization of $R \leftrightarrow 1 / R$ for the $2 \mathrm{D}$ case. Again this is a symmetry as long as we also transform momenta $m_{1}, m_{2}$ with winding $n_{1}, n_{2}$. The field $T$ is then at the origin of the name $T$ - duality (but the field $U$ is unrelated with the so-called $U$-duality). The third symmetry exchanges the complex structure $U$ with the Kähler structure $T$ and it is called 'mirror symmetry'. The moduli $U$ and $T$ each parametrize a complex plane $S L(2, \mathbb{R}) / O(2)$, the duality symmetry implies that they can only live in the fundamental domain defined by all the points of the product of complex spaces $S L(2, \mathbb{R}) / O(2) \otimes S L(2, \mathbb{R}) / O(2) \cong$ $O(2,2, \mathbb{R}) /(O(2) \times O(2))$ identified under the duality group $S L(2, \mathbb{Z})_{U} \times S L(2, \mathbb{Z})_{T}=O(2,2, \mathbb{Z})$.

This is the situation that gets generalized to higher dimensions. In general, compactification on a $d$-dimensional torus has the Narain 17 moduli space $\mathcal{M}=O(d, d, \mathbb{R}) / O(d) \times O(d)$ with points identified under the duality group $O(d, d, \mathbb{Z})$. For the heterotic string with 16 extra left moving coordinates $\mathcal{M}=O(d+$ $16, d, \mathbb{R}) / O(d+16) \times O(d)$ with a similar modification to the duality group.

A slightly more complicated example is the $2 \mathrm{D}$ black hole defined by the metric $-d u d v /(1-$ $u v)$ [18]. Diagonalizing the metric, it can be easily seen that the asymptotically flat region $u v<0$ is defined by the metric $d r^{2}-\tanh ^{2} r d t^{2}$, the region between the horizon and the singularity $0<u v<$ 1 has the metric $-d t^{2}+\tan ^{2} t d r^{2}$ whereas beyond the singularity, $u v>1$ it is $d r^{2}-\operatorname{coth}^{2} r d t^{2}$. The geometry is selfdual, but the asymptotically flat region and the region beyond the singularity are mapped to each other (global symmetry, $t \rightarrow t+$ constant implies $\left.\tilde{G_{t t}}=1 / G_{t t}\right)$. Whereas the region between the singularity and horizon is selfdual, since $\cot t=\tan (t-\pi / 2)$. This is remarkable because, in particular, duality is exchanging singularity $\leftrightarrow$ horizon [19]. Moreover, in simple extensions of this geometry to 3D, the geometry is a black string (in the sense that the singularity is not point like but one-dimensional) [20], 15] which is no longer selfdual. It is dual to another black string where now the singularity of one geometry is mapped to a regular surface in the asymptotically flat region of the other geometry and on which, contrary to the horizon, classical computations are dominant. This raises the hope that singularities may be consistently treated in string theory by performing calculations in the dual geometry.

It is then interesting to investigate if these properties hold for physically more interesting objects such as $4 \mathrm{D}$ black holes. In particular the Schwarzschild metric

$d s^{2}=-(1-2 M / r) d t^{2}+\frac{d r^{2}}{1-2 M / r}+r^{2} d \Omega$

times any CFT with $c=22$ is a classical solution of bosonic string theory to leading order in $\alpha^{\prime}$. Here $d \Omega=\left(d \theta^{2}+\sin ^{2} \theta d \phi^{2}\right)$ is the element of solid angle. Direct application of the standard duality transformation to this metric for time translations, gives the dual metric

$d \tilde{s}^{2}=-\frac{d t^{2}}{1-2 M / r}+\frac{d r^{2}}{1-2 M / r}+r^{2} d \Omega$

with the dilaton field now given by $\tilde{\Phi}=\Phi-$ $\log (1-2 M / r)$. This metric defines a geometry with naked singularities at $r=0$ and $r=2 M$, as it can be verified by computing the curvature scalar $R=\frac{4 M^{2}}{(2 M-r) r^{3}}$. It is easy to check that the string background equations are satisfied by the dual metric and dilaton $\tilde{\Phi}$. We have then found a spherically symmetric solution of the string background equations, which is not a black hole, but has naked singularities and is dual to the Schwarzschild solution.

A similar analysis can be done for the $4 \mathrm{D}$ charged dilatonic black hole. In this case the met- 
ric is 21

$$
\begin{aligned}
d s^{2}= & -\frac{(1-2 M / r)}{\left(1-Q^{2} / M r\right)} d t^{2} \\
& +\frac{d r^{2}}{(1-2 M / r)\left(1-Q^{2} / M r\right)}+r^{2} d \Omega,
\end{aligned}
$$

the dilaton field is $\Phi=-\log \left(1-Q^{2} / M r\right)$ and the electric field $F_{t r}=e^{\Phi} Q /\left(2 r^{2}\right)$. It is very interesting to note that the dual of this solution with respect to time translations gives exactly the same solution except that the mass parameter $M$ changes into $Q^{2} / 2 M$, therefore it relates the black hole domain $Q^{2}<2 M^{2}$ to the naked singularity domain $Q^{2}>2 M^{2}$. In particular, the extremal solution $Q^{2}=2 M$ is selfdual.

Extracting physical implications to these results in $4 \mathrm{D}$ is premature because these solutions are only classical solutions of the leading order string background equations and the physically interesting regions where the horizons and singularities are, usually correspond to strong coupling string theory where perturbative calculations are not relevant. For the exact solutions not that much can be said but it can be shown that $T$-duality associated with time translations in a static spherically symmetric string background, exchanges the ADM mass with the dilaton charge and the axion charge with the Taub-NUT parameter. Furthermore, as it will be seen in other lectures at this conference, the existence of $S$-duality allows to understand better the strong coupling regions of these geometries, leading to the impressive calculation of the black-hole entropy/area relation from the counting of microscopic states.

Before finishing this subsection it is worth remarking some general properties that have been found for abelian $T$-duality. First it was shown by Roček and Verlinde that if the coordinate associated with the abelian isometry is periodic, then the two dual theories define the same conformal field theory, if the coordinate is not periodic it is not established (for a discussion of this issue see for example 22] and [23] ). Also in 9] and 22], global issues of abelian $T$ duality where discussed, in particular by considering the $2 \mathrm{D}$ surface to be a torus, it can be explicitly seen that the periodicity of the dual variable coincides with the periodicity of the original coordinate and duality survives at any order in string perturbation theory. Furthermore, it was shown that $T$-duality is a remnant of a gauge symmetry [24] in the sense that at the self dual point there is an enhanced gauge symmetry since the Kaluza-Klein $U(1)$ symmetry of circle compactification gets enhanced to $S U(2)$ at the selfdual radius $R=\sqrt{\alpha^{\prime}}$ (take $m=n= \pm 1$ and $N_{R}=1$ in equation (4) showing the existence of extra massless bosons which complete $S U(2))$. This symmetry is broken at any other point leaving duality as the discrete unbroken symmetry. This has been used to argue that $T$ - duality should also survive nonperturbative string effects. The $O(d, d)$ symmetries of toroidal compactifications can be generalized to any background with $d$ abelian isometries [25]. Finally the explicit form of the duality transformation given above may be corrected by considering higher order terms in the sigma model expansion parameter $\alpha^{\prime}$, see for instance [26]. This has raised the interesting question of combining duality with the renormalization group flow in the sense that the beta function may transform in a well defined way under duality and could give information about the phase structure of the theory.

\subsection{General Antisymmetric Tensors}

The discussion of abelian $T$-duality can be immediately generalized to arbitrary antisymmetric tensors of rank $h-1, B_{h-1}$ in $D$ dimensions [27]. In this case the simplest action is

$S=\int d^{D} x\left(\frac{1}{g^{2}}\left(\partial_{M_{1}} B_{M_{2} \cdots M_{h}}\right)^{2}+\cdots\right)$

The action is invariant under the shift of $A$ by an arbitrary closed form. $\delta B_{h-1}=d \Omega_{h-1}+K_{h}$ with $K_{h}$ an arbitrary harmonic form. The symmetry here is more general than the $2 \mathrm{D}$ case because it includes a local symmetry defined by $d \Omega$ and a global symmetry given by the shift in $K$. This is the global symmetry we need for dualization. Notice that gauging this symmetry involves a gauge field which is an antisymmetric tensor of rank $h$, $A_{h}$. Again we can dualize in two equivalent ways. First we construct a first order action by substituting $d B$ in the action by $A_{h}$ and imposing the constraint $d A_{h}=0$ by adding a Lagrange multi- 
plier $\Lambda_{D-h-1}$.

$S_{G}=\int d^{D} x\left(\frac{1}{g^{2}}\left(A_{h}\right)^{2}+\Lambda_{D-h-1} \cdot\left(d A_{h}\right)\right)$.

Where for simplicity we have defined $\Lambda_{D-h-1}$. $\left(d A_{h}\right)=\epsilon^{M_{1} \cdots M_{D}} \partial_{M_{2}} A_{M_{2} \cdots \Lambda_{h+1}} \Lambda_{M_{h+2} \cdots M_{D}}$. Integrating $\Lambda_{D-h-1}$ reproduces the original action whereas integrating $A_{h}$ leads to the dual action

$S=\int d^{D} x\left(g^{2}\left(\partial_{M_{1}} \Lambda_{M_{2} \cdots \Lambda_{D-h}}\right)^{2}+\cdots\right)$.

This is the higher dimensional generalization of the $R \leftrightarrow 1 / R$ duality of strings on a circle. To make the analogy complete we are considering compact antisymmetric tensor field theories for which the gauge symmetry is $U(1)$ rather than the real line 28. Notice that, as in the $2 \mathrm{D}$ case, the coupling constant $g$ could have been absorbed in the definition of the fields entering then in the periodicity of the fields. In that case we can see that the two dual variables are related by a Poincaré duality: $d \Lambda={ }^{*} d B$. And duality exchanges field equations with Bianchi identities. For the particular case of 4D QED, this implies that duality exchanges electric and magnetic fields which is the original duality of Maxwell equations in vacuum mentioned in the introduction. Again, as in the $2 \mathrm{D}$ case, we can see $A_{h}$ as the gauge field of the global symmetry and the Lagrange multiplier term as imposing vanishing field strength. Also to be more strict we should have introduced external current couplings in the action so that the path integral would be a function of that current as in equation (5). Duality then holds for any correlation function. Similar to the $2 \mathrm{D}$ case where the dilaton transformation was imposed, if we consider topologically nontrivial spacetimes there would be an extra term proportional to the Euler number of the manifold [29], generalizing the dilaton transformation in the $2 \mathrm{D}$ case. We will not consider further this possibility.

Finally, as a gauge field naturally couples to a pointlike charged particle by the coupling $\int A_{\mu} d x^{\mu}$, the field $B_{h-1}$ naturally couples to an $h-2$ dimensional object, a $p$-brane $(p=h-2)$ which is the analogous of the electric charges in QED. The dual field $\tilde{B}_{d-h} \equiv \Lambda_{D-h-1}$ couples to a magnetic $D-h-2$ dimensional brane analogous to the magnetic monopole. We will then see in section 4 that the duality between electric charges and magnetic monopoles is generalized to a duality between electric $(h-2)$-branes and magnetic $(D-h-2)$-branes 30 .

\subsection{Abelian Bosonization}

Now that we have a definite prescription for duality we can apply it to any system with a global symmetry. The simplest example that comes to mind is a free fermion in $2 \mathrm{D}$ with the Dirac action

$S_{F}=\int d^{2} x\left(-\bar{\psi} \not \partial \psi+i J_{\mu} \bar{\psi} \gamma^{\mu} \psi\right)$

where we introduced explicitly the external source $J_{\mu}$ to keep track of the functional dependence of the partition function $Z[J]=\int \mathcal{D} \psi e^{i S_{F}}$. Notice that this action has the global symmetry $\psi \rightarrow e^{i \theta} \psi$, therefore we can just follow the same prescription as above, $i e$ we gauge the global symmetry and impose the constraint of vanishing gauge field strength [31]. (The first order formalism used before does not apply clearly in this case.) We have then to consider the partition function

$Z_{G}[J] \equiv \int \mathcal{D} \psi \mathcal{D} A_{\mu} \mathcal{D} \Lambda e^{i S_{G}\left(\psi, J_{\mu}+A_{\mu}, \Lambda\right)}$

where

$S_{G} \equiv \int d^{2} x\left(\mathcal{L}_{F}\left(\psi, J_{\mu}+A_{\mu}\right)+\frac{1}{2} \Lambda \varepsilon^{\mu \nu} F_{\mu \nu}\right)$

Since we have to fix the gauge symmetry, it is convenient to work in the Lorentz gauge $\partial \cdot A=0$. It is straightforward to see that integrating the Lagrange multiplier $\Lambda$ sets $F=0$ which together with the Lorentz gauge allows us to set $A_{\mu}=0$, recovering the original action. In order to find the dual action we have to integrate first over $\psi$ and $A_{\mu}$ and find the effective action $S_{B}[\Lambda, J]$. This can be performed thanks to the well known calculation of Schwinger:

$$
\begin{aligned}
& \int \mathcal{D} \psi \exp \left[i \int d^{2} x\left(-\bar{\psi} \not \partial \psi+i \bar{\psi} \gamma^{\mu} \psi A_{\mu}\right)\right] \\
= & \exp \left[\frac{i}{4 \pi} \int d^{2} x F^{\mu \nu} \square^{-1} F_{\mu \nu}\right] .
\end{aligned}
$$

Next we have to integrate over $A_{\mu}$. The Lorentz gauge implies we can write $A_{\mu}=\varepsilon_{\mu \nu} \partial^{\nu} \varphi$, the corresponding jacobian is a constant and the integral 
over $\varphi$ is gaussian. We end up with the bosonic lagrangian:

$\mathcal{L}_{B}(\phi, J)=-\frac{1}{2} \partial_{\mu} \phi \partial^{\mu} \phi+\frac{1}{\sqrt{\pi}} \varepsilon^{\mu \nu} \partial_{\mu} \phi J_{\nu}$

where we have defined $\phi \equiv \sqrt{\pi} \Lambda$. Therefore we have shown that the actions for free fermions and free bosons are dual to each other, reproducing the standard bosonization result in a fashion that makes bosonization and standard dualities be recognized as two manifestations of the same effect. By comparing the coefficients of the source $J_{\mu}$ in both actions we can explicitly see the equivalence:

$i \bar{\psi} \gamma^{\mu} \psi \leftrightarrow-\frac{1}{\sqrt{\pi}} \varepsilon^{\mu \nu} \partial_{\nu} \phi$

which is the standard statement of bosonization. Coupling the axial current to another source gives the mapping $i \bar{\psi} \gamma^{\mu} \gamma_{3} \psi \leftrightarrow \frac{1}{\sqrt{\pi}} \partial^{\nu} \phi$.

Notice that a crucial step in our derivation of the bosonic action was the use of the explicit calculation of the fermion determinant by Schwinger. For a more complicated fermionic theory, including four-fermion couplings and mass terms there is no explicit calculation of the determinant but we still can use an extra property of the system, this is that besides the vectorlike phase symmetry we used for dualization, there is also an axial symmetry of the fermionic theory: $\psi \rightarrow \exp \left(i \alpha \gamma_{3}\right) \psi$. This symmetry is anomalous but using the gauged action with the Lagrange multiplier constraint above we can see that the $\Lambda F$ term plays the role of a Wess-Zumino anomaly cancelling term. This can be seen explicitly by realizing that the anomaly can be canceled by asigning a transformation to $\Lambda$ under axial transformation $\Lambda \rightarrow \Lambda+\alpha / \pi$. This combined with the fact that the integration over the gauge field gives a delta function forcing the identifications (23) in terms of expectation values, is enough to determine that the dual action is given by (22), without the need of using the explicit fermion determinant. A similar argument can be used to prove that the massive Thirring model

$S=S_{F}+\int d^{2} x\left(\frac{1}{2} g^{2}\left(\bar{\psi} \gamma^{\mu} \psi\right)\left(\bar{\psi} \gamma_{\mu} \psi\right)-m \bar{\psi} \psi\right)$ is dual to the sine-Gordon model

$S_{S G}=S_{B}-A m \int d^{2} x(1-\cos \beta \phi)$.

With $A$ an undetermined constant and $\beta$ is the coupling given by $\beta=2 \pi / \sqrt{\pi+g^{2}}$. We can then see that a weak coupling Thirring model corresponds to a strongly coupled sine-Gordon model and viceversa. This then reproduces the well known results on bosonization.

The power of the present approach relies on the fact that it provides a unified way to treat bosonization and duality, something that was not done in the past. Furthermore, it allows to extend the concept of bosonization to any dimension $D \geq 2$ [32]. In an arbitrary dimension we can immediately see that the bosonic coordinate $\Lambda$ will be extended from a scalar in $2 \mathrm{D}$ to an antisymmetric tensor of rank $D-2$ in order to have the Lagrange multiplier term in the Lagrangian $\varepsilon_{M_{1} \cdots M_{D}} F^{M_{1} M_{2}} \Lambda^{M_{3} \cdots M_{D}} \equiv \Lambda \cdot d A$.

$\mathcal{L}_{G}=\mathcal{L}_{F}+m \bar{\psi} \psi+i \bar{\psi} \gamma^{\mu} \psi A_{\mu}+(\Lambda \cdot d A)$

The problem we face in higher dimensions is that the fermion determinant is not computed, the symmetry argument we used does not help in higher dimensions mainly because of the properties of the $\gamma$ matrices. We should then rely on special cases. A simple case is to consider a massive fermion in the limit of large mass. For this case

$$
\begin{aligned}
& \int \mathcal{D} \psi \exp \left\{-i \int d^{D} x \bar{\psi}\left[\gamma^{\mu} D_{\mu}+m\right] \psi\right\} \\
= & \exp \left\{\frac{i}{2} \int d^{D} x A_{\mu} \Pi_{D}^{\mu \nu} A_{\nu}+\cdots\right\},
\end{aligned}
$$

Where $D_{\mu} \equiv\left(\partial_{\mu}-i A_{\mu}\right)$. The vacuum polarization $\Pi_{D}^{\mu \nu}$ has been computed in the past in a $1 / m$ expansion, the first order term is:

$$
\begin{aligned}
\Pi_{D}^{\mu \nu} & =k_{D}\left(\square \eta^{\mu \nu}-\partial^{\mu} \partial^{\nu}\right), \quad D>3 \\
& =k_{3} \varepsilon^{\mu \nu \lambda} \partial_{\lambda} \quad D=3
\end{aligned}
$$

with $k_{D}$ a $D$-dependent constant and $k_{3}=$ sign $m / 8 \pi^{2}$. For $D>3$ this leads to the nonlocal action:

$S=-\int \mathrm{d}^{d} x\left(\frac{1}{2 k_{D}} \Omega_{\mu} \frac{1}{\square} \Omega^{\mu}+\Omega_{\mu} J^{\mu}\right)$. 
where $\Omega \equiv \tilde{\Lambda}$. For $D=3$ however, we get a local action of the Chern-Simons type

$$
S=-\int d^{3} x \varepsilon^{\mu \nu \rho}\left(\frac{1}{2 k_{3}} \Lambda_{\mu} \partial_{\nu} \Lambda_{\rho}+J_{\mu} \partial_{\nu} \Lambda_{\rho} .\right)
$$

with the standard bosonization relations

$i \bar{\psi} \gamma^{\mu} \psi \leftrightarrow-\varepsilon^{\mu \nu \rho} \partial_{\nu} \Lambda_{\rho}$

\section{NON-ABELIAN DUALITY}

In this section we will try to generalize the results of the previous section for cases when the global symmetry is non-abelian.

\subsection{Non-Abelian $T$-Duality}

Consider the $\sigma$-model action (1) and assume that the target space metric has a group $\mathcal{G}$ of non-abelian isometries [10], [33, [34], [35]. In this case, $Q_{M N}$ does depend on $X^{m}$ and transforms accordingly under $X^{m} \rightarrow g_{n}^{m} X^{n}, g \in \mathcal{G}$. We gauge the symmetry corresponding to a subgroup $H \subseteq \mathcal{G} \partial X^{m} \rightarrow D X^{m}=\partial X^{m}+A^{\alpha}\left(T_{\alpha}\right)_{n}^{m} X^{n}$, and add to the action the term $\int d^{2} z \operatorname{tr}(\Lambda F)=$ $\int d^{2} z \Lambda_{\alpha} F^{\alpha}$, where in this case the gauge field strength is, in matrix notation, $F=\partial \bar{A}-\bar{\partial} A+$ $[A, \bar{A}]$. The $N \times N$ matrices $T_{\alpha}$ form an adjoint representation of the group $H$. In the path integral we have to consider

$$
\begin{aligned}
\mathcal{Z}= & \int \frac{\mathcal{D} X}{V_{\mathcal{G}}} \int D \Lambda D A D \bar{A} \\
& e^{\left\{-i\left(S_{G}[X, A, \bar{A}]+\int d^{2} z \operatorname{tr}(\Lambda F)\right)\right\}},
\end{aligned}
$$

where $V_{\mathcal{G}}$ is the "volume" of the group of isometries and $\mathcal{D} X$ is the measure that gives the correct volume element $\mathcal{D} X=D X \sqrt{G} e^{-\Phi}$. Similar to the abelian case, the original action is obtained by integrating out the Lagrange multiplier $\Lambda$. Locally, this forces the gauge field to be pure gauge $A=h^{-1} \partial h, \bar{A}=h^{-1} \bar{\partial} h, h \in H$. By fixing the gauge with the choice $A=0, \bar{A}=0$ we reproduce the original theory. The dual theory is obtained by integrating out the gauge fields in the path integral (32). Integrating over the gauge fields $A$, $\bar{A}$ we obtain

$$
\int \mathcal{D} X D \Lambda \delta[\mathcal{F}] \operatorname{det} \frac{\delta \mathcal{F}}{\delta \omega} e^{-i S^{\prime}[X, \Lambda]} \operatorname{det}\left(f^{-1}\right)
$$

where $\mathcal{F}$ is the gauge fixing function and $\omega$ are the parameters of the group of isometries. The matrix $f$ is the coefficient of the quadratic term in the gauge fields and $\tilde{S}$ is given by

$$
\tilde{S}[X, \Lambda]=S[X]-\frac{1}{4 \pi \alpha^{\prime}} \int d^{2} z \bar{h}_{\alpha}\left(f^{-1}\right)^{\alpha \beta} h_{\beta}
$$

Here $h$ and $\bar{h}$ are the currents coupled to $\bar{A}$ and $A$ respectively [10]. After the gauge fixing, denoting the new coordinates in the dual manifold collectively by $Y$, we have

$\mathcal{P}=\int \mathcal{D} Y e^{-i \tilde{S}[Y]} \operatorname{det}\left(f(Y)^{-1}\right)$.

The Fadeev-Popov determinant in the path integral contributes to the measure such that the correct volume element for the dual manifold is obtained $\mathcal{D} Y=D Y \sqrt{\tilde{G}} e^{-\Phi^{\prime}}$. The factor $\operatorname{det}\left(f^{-1}\right)$ in the partition function can be computed using standard regularization techniques [8]. It generates a new local term in the action of the form $\frac{1}{8 \pi \alpha^{\prime}} \int d^{2} z \alpha^{\prime} R^{(2)}(\Delta \Phi)$, which corresponds to the change in the dilaton due to the duality transformation

$\tilde{\Phi}=\Phi-\log \operatorname{det} f$.

This change in the dilaton transformation is the shift necessary to retain the conformal invariance of the dual theory.

In general, we cannot write explicitly the gauge fixed dual action. Therefore, we are not able to present the new metric and antisymmetric tensor fields in a closed form, as was done for the abelian case in equations (3). As an example, let us consider a theory for which the target space metric has a maximally symmetric subspace with $\mathcal{G}=S O(3)$ and no antisymmetric tensor. The coordinates $X^{M}, M=1, \ldots, D$, can be decomposed into the two angular coordinates $(\theta, \phi)$ describing 2-dimensional spheres, and $D-2$ extra coordinates $\left(v^{\mu}\right)$ specifying the different spheres in the $D$ dimensional spacetime. The metric can then be decomposed in the form

$S=S[v]+\int d^{2} z a^{2} \Omega\left(\partial \theta \bar{\partial} \theta+\sin ^{2} \theta \partial \varphi \bar{\partial} \varphi\right)$

It is more convenient to treat the coordinates $\theta$ and $\phi$ in terms of cartesian coordinates $X^{m}$ in 
3-dimensional space on which $S O(3)$ can act linearly, so we write the $\sigma$-model action in the form

$$
\begin{aligned}
S= & S[v]+\int d^{2} z \Omega(v)\left\{G_{m n} \partial X^{m} \bar{\partial} X^{n}+\right. \\
& \left.\frac{1}{2 a \sqrt{\Omega}} \lambda\left(G_{m n} X^{m} X^{n}-a^{2}\right)\right\},
\end{aligned}
$$

where $S[v]=\int d^{2} z G_{\mu \nu}(v) \partial v^{\mu} \bar{\partial} v^{\nu}$, the metric $G_{m n}$ is diagonal and constant and the Lagrange multiplier term fixes the 3 dimensional space to be a sphere of radius $a$. Gauging this action and fixing the gauge $A=\bar{A}=0$ we obtain the original action. Another convenient choice of gauge is to set $X^{1}=X^{2}=0, \quad X^{3}=a, A^{1}=\partial \theta$, $A^{2}=-\sin \theta \partial \varphi$ and $A^{3}=\cos \theta \partial \varphi$ leading to the original action in spherical coordinates .

We can write a general expression for the dual action after fixing the coordinates $X^{m}$ as above, but before fixing the remaining degree of freedom corresponding to one of the Lagrange multipliers $\Lambda_{\alpha}$. We then have (from here on, we ommit writing explicitly the dilaton term of equation (1))

$\tilde{S}=S[v]+\frac{1}{4 \pi \alpha^{\prime}} \int d^{2} z\left(\partial \Lambda_{\alpha}\left(f^{-1}\right)^{\alpha \beta} \bar{\partial} \Lambda_{\beta}\right)$

From this expression, we can in principle read off the new background fields as in (3). This is actually the general expression for any group. But we still have to complete the gauge fixing for the $\Lambda_{\alpha}$. In our case, choosing $\Lambda_{2}=0$ and defining $x^{2}=\Lambda_{1}^{2}+\Lambda_{3}^{2}$ and $y=\Lambda_{3}$, we obtain the dual theory action

$\tilde{S}=S[v]+\int d^{2} z \frac{\left(a^{4} \Omega(v)^{2} \partial y \bar{\partial} y+x^{2} \partial x \bar{\partial} x\right)}{4 \pi \alpha^{\prime} a^{2} \Omega(v)\left(x^{2}-y^{2}\right)}$

and the dual dilaton field is now given by $\tilde{\Phi}=$ $\Phi-\log \left[a^{2} \Omega(v)\left(x^{2}-y^{2}\right)\right]$.

Consider the dual geometry of Schwarschild with respect to the $S O(3)$ symmetry. We find

$$
\begin{aligned}
d s^{2}= & -(1-2 M / r) d t^{2}+\frac{d r^{2}}{1-2 M / r} \\
& +\frac{1}{r^{2}\left(x^{2}-y^{2}\right)}\left[r^{4} d y^{2}+x^{2} d x^{2}\right],
\end{aligned}
$$

with the new dilaton $\tilde{\Phi}=\Phi-\log \left[r^{2}\left(x^{2}-y^{2}\right)\right]$. The regions $x=y$ and $r=0$ are real singularities whereas $r=2 M$ is only a metric singularity corresponding to a horizon as in the original case. Notice that the metric is not spherically symmetric, in fact it does not have non-abelian isometries, raising the question of how to dualize back, this is a definite difference with the abelian case and shows in some way that the global symmetry is not an essential ingredient to obtain duality. We will see that Poisson-Lie $T$-duality takes care of this situation in an elegant way. Again, it is straightforward to check that this background fields satisfy the string background field equations thus providing 'new' string vacua.

To finish this subsection we can mention some general features of non-abelian duality. If the non-abelian group $\mathcal{G}$ is non-compact, there may be an anomaly that prevents the dual model from being conformal. An example of this type was considered in [34] and the understanding of the anomaly was found in [35]. In this paper it is also realized that a better treatment of non-abelian duality is to express the dual theory in terms of a group-valued coordinate $g$ instead of the algebravalued coordinate $\Lambda$, this can be achieved by performing a non-local change of variables $\partial \Lambda \rightarrow$ $g^{-1} \partial g$. this allows to formulate non-abelian duality in a way closer to the abelian case, it is also crucial for the non-abelian bosonization we will discuss next. Furthermore, it has also been shown that non-abelian duality can also be realized as a particular canonical transformation [36]. Global issues are less under control for the non-abelian case and not much progress has been achieved in this direction. Finally it is worth remarking that a higher dimensional generalization of non-abelian duality can be thought in two ways, one is work with scalar fields in higher dimensions defining a $\sigma$-model invariant under a nonabelian group. The steps to dualize are identical to what we have done and again the complication is only technical. On the other hand we could consider antisymmetric tensors of higher rank, like nonabelian gauge fields. A duality transformation has been attempted for this case which could be relevant for $4 \mathrm{D}$ QCD for instance. However, naive dualization takes a Yang-Mills gauge theory into another theory which is not Yang-Mills 37. Non-abelian antisymmetric tensor theories of rank greater than two have been argued not to 
exist 39.

\subsection{Poisson-Lie T-Duality}

A very elegant generalization of non-abelian $T$ duality is the Poisson-Lie $T$-duality introduced by Klimčík and Ševera [38]. We will briefly explain here the main idea of this generalization.

If the $\sigma$-model ( 1 ) is invariant under the action of a group $\mathcal{G}$, the corresponding 'Noether' current:

$J_{a}=v_{a}^{M} Q_{M N}\left(\partial X^{M} d z-\bar{\partial} X^{N} d \bar{z}\right)$

is conserved i.e. $d J_{a}=0$. Here $v_{a}^{M}$ is the vector field associated to the right action of $G$. If the current is not conserved but satisfy:

$d J_{a}=\frac{1}{2} \tilde{c}_{a}^{k l} J_{k} \wedge J_{l}$

where $\tilde{c}_{a}^{k l}$ are the structure constants of another, dual Lie group $\tilde{\mathcal{G}}$, then the $\sigma$-model is said to have the $\mathcal{G}$ Poisson-Lie symmetry with respect to $\tilde{\mathcal{G}}$. Then we can generalize the idea of duality based on a global symmetry to cases with Poisson-Lie symmetry. In that case we can immediately see that abelian and nonabelian dualities are just particular cases of this duality where the dual group $\tilde{\mathcal{G}}$ is abelian and so the structure constants vanish. In that case the nature of $\mathcal{G}$ distinguishes between abelian and non-abelian dualities. This explains the lack of non-abelian isometries in the models considered in the previous subsection. For non-abelian $\tilde{\mathcal{G}}$ this is a new duality symmetry. Therefore Poisson-Lie T-duality is a more general setting to formulate $T$-duality. Duality here is manifest since the role of the two dual groups $\mathcal{G}, \tilde{\mathcal{G}}$ can be interchanged. These groups form what is called a Drinfeld double, defined by the decomposition of a bigger group $\mathcal{D}$ into $\mathcal{D}=\mathcal{G}+\tilde{\mathcal{G}}$ where the algebras of $\mathcal{G}, \tilde{\mathcal{G}}$ are maximally isotropic subalgebras of that of $\mathcal{D}$ with respect to a nondegenerate invariant bilinear form of the algebra of $\mathcal{D}$. Using this approach it has been possible to generalize some of the properties of abelian duality such as the momentum/winding exchange of the two dual models.

\subsection{Non-abelian Bosonization}

In the same way that abelian bosonizaton was understood in section 2.3 as a particular case of abelian duality, we can also understand nonabelian bosonization as a particular case of nonabelian duality 40. The starting point is a system of Majorana fermions in $1+1$ dimensions, invariant under the global symmetry $O(N)$. The partition function is

$\mathcal{Z}=\int \mathcal{D} \psi \exp \left(-\frac{i}{2} \int d^{2} x \bar{\psi} \gamma^{\mu}\left(\partial_{\mu}-a_{\mu}\right) \psi\right)$

with $a_{\mu}$ being a matrix valued external fields. Again dualization can be ahieved by gauging the anomaly free $O(N)$ group and imposing the corresponding field strength to vanish.

$\mathcal{Z}=\int \mathcal{D} \psi \mathcal{D} A \mathcal{D} \Lambda e^{i \int d^{2} x\left(-\frac{1}{2} \bar{\psi} \gamma^{\mu} D_{\mu} \psi+\varepsilon^{\mu \nu} \Lambda F_{\mu \nu}\right)}$

with the covariant derivative $D_{\mu} \equiv \partial_{\mu}-i\left(a_{\mu}+\right.$ $A_{\mu}$ ). Fixing the gauge and performing the fermion integral will lead a theory in terms of $\Lambda$ which is the desired bosonization. Luckily, as in the abelian case, the fermion integral has been already computed by Polyakov and Wiegmann [41]. The result is better expressed by redefining $A_{\mu}$ and $a_{\mu}$ in terms of group valued variables $\left(a_{ \pm}=i h_{ \pm}^{\dagger} \partial_{ \pm} h_{ \pm}\right.$etc. $)$.

$\mathcal{Z}[a]=\mathcal{Z}[a=0] \exp \left(-i \Gamma\left[h_{-} h_{+}^{\dagger}\right]\right)$

With $\Gamma$ the Wess-Zumino-Witten action 41, 4 . Using this and performing a nonlocal change of variables for the algebra-valued Lagrange multiplier $\partial \Lambda \leftrightarrow g^{\dagger} \partial g$, we find that the partition function is 40

$\mathcal{Z}_{G}[a]=\int \mathcal{D} g \exp i \Gamma_{G W Z W}[g, a]$

With $\Gamma_{G W Z W}$ the gauged Wess-Zumino-Witten action $\Gamma_{G W Z W}[g, a] \equiv \Gamma\left[h_{-} g h_{+}^{\dagger}\right]-\Gamma\left[h_{-} h_{+}^{\dagger}\right]$. We have then seen that the bosonized action is the level one WZW action dual to the action for free Majorana fermions. By comparing the couplings to the external field $a_{\mu}$ we can also get the standard bosonization relations

$i \bar{\psi} \gamma_{-} \psi \leftrightarrow \frac{i}{2 \pi} g^{\dagger} \partial_{-} g, \quad i \bar{\psi} \gamma_{+} \psi \leftrightarrow-\frac{i}{2 \pi} \partial_{+} g g^{\dagger}$ 


\section{BROKEN GLOBAL SYMMETRIES}

In this section we will concentrate on the general antisymmetric tensor field theories discussed in section 2 and abelian duality. The question we will pose is if the existence of the global symmetry is not only a sufficient but also necessary condition for duality. The answer is no. A way to illustrate this is by starting with a system with a global symmetry and break it by the existence of topological defects. Then we can ask what happens to duality. We will see that duality not only survives this breaking but gets enriched in the sense that it relates different phases of the theory.

\subsection{QED and monopoles}

Let us start with the best known case of duality which is Maxwell's equations. We know that in vacuum these equations are invariant under the exchange of electric and magnetic fields. Duality exchanges the two Bianchi identities with the two field equations by exchanging the field strength $F_{\mu \nu}$ with its dual $\tilde{F}_{\mu \nu} \equiv \varepsilon_{\mu \nu \rho \sigma} F^{\rho \sigma}$. Duality was easily derived in section 2 . However this was true only for the case of Maxwell's equations without sources. In order to generalize this duality to the case with sources we have to follow Dirac and include electric and magnetic sources. The simplest case is the source due to the presence of a 'elementary' electric charge $q_{e}$ and a 'solitonic' magnetic charge $q_{m}$ for which the corresponding currents are:

$J_{e, m}^{\mu}=q_{e, m} \int d \xi^{\mu} \delta^{4}(x-\xi)$.

In order to derive duality for this case we first need to write an action that incorporates both sources. Inclusion of the elctric source is straightforward, we only need to add a term of the form $A_{\mu} J_{e}^{\mu}$ to the Maxwell action. The magnetic current is not that simple but it can be done by first noticing that in the presence of a magnetic monopole the field strength is no longer of the form $F_{\mu \nu}=\partial_{\mu} A \nu-\partial_{\nu} A_{\mu}$. In fact the magnetic field due to a monopole can be written as

$$
\vec{B}=\frac{q_{m} \hat{r}}{4 \pi r^{2}}=\nabla \times \vec{A}-q_{m} \theta(-z) \delta(x) \delta(y) \hat{z}
$$

where the last term represents the Dirac string along the $-z$ axis. Writing this in a covariant form implies

$F_{\mu \nu}=\left(\partial_{\mu} A_{\nu}-\partial_{n} u A_{\mu}\right)-V_{\mu \nu}$

with $V_{\mu \nu} \equiv q_{m} \varepsilon_{\mu \nu \rho \sigma} \int \delta^{4}(x-\xi) d \xi^{\rho} d \xi^{\sigma}$, from which we can easily see that $\partial_{\nu} \varepsilon^{\nu \mu \rho \sigma} V_{\rho \sigma}=J_{m}^{\mu}$. Therefore we can write the action as:

$S=\int d^{4} x\left(F_{\mu \nu}^{2}+A_{\mu} J_{e}^{\mu}\right)$

with $F_{\mu \nu}$ as given above. The first observation we can make is that the field equations derived from this action are precisely Maxwell's equations in the presence of the electric and magnetic sources:

$\partial_{\nu} F^{\mu \nu}=J_{e}^{\mu} \quad, \quad \partial_{\nu} \tilde{F}^{\mu \nu}=J_{m}^{\mu}$.

Duality is not trivial for this action since tere is no longer the global symmetry shifting the field $A_{\mu}$. Nevertheless, knowing how $F_{\mu \nu}$ differs from a total derivative allows us to still be able to dualize this system. In order to find the dual action, we start with the first order Lagrangian (with $F_{\mu \nu}$ arbitrary)

$\mathcal{L}=\left(\left(F_{\mu \nu}-V_{\mu \nu}\right)^{2}+\tilde{F}_{\rho \sigma}\left(\partial_{\rho} \Lambda_{\sigma}-\tilde{V}_{\rho \sigma}\right)\right)$

Where $\tilde{V}_{\rho \sigma}$ satisfies $\partial_{\nu} \tilde{V}^{\mu \nu}=J_{e}^{\mu}$. From this we can see that integrating out $\Lambda_{\sigma}$ implies $F_{\mu \nu}=$ $\partial_{\mu} A_{\nu}-\partial_{\nu} A_{\mu}$ and we get back the original action. Changing the order of integration, we can integrate $F_{\mu \nu}$ and get the dual Lagrangian with $\Lambda$ as the fundamental variable:

$\tilde{\mathcal{L}}=\left(\left(\partial_{\mu} \Lambda_{\nu}-\partial_{\nu} \Lambda_{\mu}-\tilde{V}_{\mu \nu}\right)^{2}-\Lambda_{\mu} J_{m}^{\mu}\right)$

where we have used that $\int d^{4} x \varepsilon^{\mu \nu \rho \sigma} V_{\mu \nu} \tilde{V}_{\rho \sigma}=$ $q_{e} q_{m} I=2 \pi n$ where $I$ is the integer intersection number of the surfaces defined by $V_{\mu \nu}$ and $\tilde{V}_{\rho \sigma}$ and we have used the Dirac quantization condition $q_{e} q_{m}=2 \pi$ integer. From this pair of dual actions we can explicitly see the realization of electric/magnetic duality even in the presence of monopole sources that break the global symmetry. A generalization to any dimension and arbitrary antisymmetric tensors is straightforward. 


\subsection{Monopole Condensation}

We saw in the previous subsection how duality survived in the presence of electric and magnetic monopoles. Now we can imagine what happens when we have a continuous distribution of magnetic monopoles, i.e. monopole condensation. In this case the field $F_{\mu \nu}$ is not the derivative of $A_{\mu}$ not only at the Dirac string of a single monopole but for all spacetime points. Therefore in this situation it is natural to assume $F_{\mu \nu}$ as the fundamental field and write an effective action for it. Imposing that this action be local, Poincaré invariant, with no more than two derivatives and that it reduces to the Maxwell action in the limit of vanishing density of monopoles $\rho$, it uniquely fixes the action to be:

$S_{\text {conf. }}=\int d^{4} x\left(-\frac{1}{e^{2}} F_{\mu \nu}^{2}+\frac{1}{\rho^{2}}\left(\partial_{\mu} F_{\nu \alpha}\right)^{2}\right)$

we can easily see that if the monopole density vanishes only the configurations for which $\left(\partial_{\mu} F_{\nu \alpha}\right)=$ 0 contribute to the path integral implying $F=d A$ and reducing to the Maxwell action. This action describes a massive 2-index antisymmetric tensor field with mass $M=\rho / e$. We have added the subscript conf to the action since we will see it will describe the confining phase of QED.

At this point it is natural to ask whether this action has a dual and if there is a way to find it. Notice that at this level there are no traces of global symmetries. Nevertheless we can start with the first order action:

$S=\int d^{4} x\left(\frac{-1}{e^{2}} F_{\mu \nu}^{2}-\varepsilon^{\mu \nu \rho \sigma} \tilde{B}_{\mu} \partial_{\nu} F_{\rho \sigma}-\rho^{2} \tilde{B}_{\mu}^{2}\right)$

There is no Lagrange multiplier appearing linearly in this action, but the fields $\tilde{B}_{\mu}$ and $F_{\mu \nu}$ appear quadratically and therefore we can still integrate them out by means of a gaussian integration. Integrating out $\tilde{B}_{\mu}$ implies $\rho^{2} \tilde{B}_{\mu}=$ $\varepsilon_{\mu \nu \rho \sigma} \partial^{\nu} F^{\rho \sigma}$ and substituting back into the action reproduces the original theory $S_{\text {conf }}$. Integrating out $F_{\mu \nu}$ instead, leads to the dual action:

$\tilde{S}_{\text {conf }}=\int d^{4} x\left(-e^{2}\left(\partial_{\nu} \tilde{B}_{\mu}\right)^{2}+\rho^{2}\left(\tilde{B}_{\mu}\right)^{2}\right)$

This action describes a massive vector having one propagating degree of freedom with mass
$M=\rho / e$. Therefore we can see that duality survives the breaking of the global symmetry, but now is a completely different duality. Instead of relating two massless vectors as in the original (Coulomb) phase, it is now a duality between a massive 2-index field and a massive vector, both describing the confining phase of compact QED after monopole condensation. Since in the Coulomb action, duality there was a symmetry between electric and magnetic degrees of freedom, we can ask if starting from the dual Coulomb action, we could consider the condensation of electric monopoles giving rise not to a confining phase but to a 'Higgs' phase. The process is identical to the confining case because the two dual actions for the Coulomb phase were the same (except for the change of $e \leftrightarrow 2 / e$ ). Therefore we get two dual actions for the Higgs phase also describing three massive degrees of freedom in terms of a massive vector or massive two-form, with mass $\tilde{M}=\tilde{\rho} e$. We can then see that the Higgs and confining phases are identical in this case after exchanging $\tilde{\rho} \leftrightarrow \rho$ and $e \leftrightarrow 2 / e$. This is known as Higgs/confinement duality.

\subsection{Generalizations}

Now we can generalize these results to any dimension as in section (2.2) [42], 16]. In $D$ dimensions we know the Coulomb phase is described by two dual actions in terms of antisymmetric tensors $B_{h-1}$ and $\Lambda_{D-h-1}$ respectively, both carrying $\left(\begin{array}{c}D-2 \\ h-1\end{array}\right)$ degrees of freedom. In that case the 'elementary' charges are $(h-2)$-branes whereas the 'solitonic' or magnetic charges are $(D-h-2)$ branes (as we have seen from other lectures, a monopole is a 0 -brane, an instanton a (-1)-brane etc.) which are the natural objects coupling to $B_{h-1}$ and $\Lambda_{D-h-1}$ respectively. The confining phase is defined by the condensation of the magnetic objects and is described by a massive antisymmetric tensor $H_{h}$ dual to another massive antisymmetric tensor $\tilde{B}_{D-h-1}$ carrying $\left(\begin{array}{c}D-1 \\ h\end{array}\right)$ degrees of freedom with mass $\rho / e$. The Higgs phase can be generated by the condensation of the elementary $(h-2)$-branes and would be described by massive antisymmetric tensors $\tilde{H}_{D-h}$ dual to $B_{h-1}$, both carrying $D-1 / h-1$ massive degrees 
Table 1

Duality for the Coulomb, Higgs and confinement phases

$$
\text { Original Lagrangian Dual Lagrangian }
$$

Coulomb phase $\quad \frac{1}{e^{2}}\left(d B_{h-1}\right)^{2} \quad \frac{e^{2}}{4}\left(d \tilde{B}_{d-h}\right)^{2}$

Confinement phase $\quad \frac{1}{\rho^{2}}\left(d H_{h}\right)^{2}+\frac{1}{e^{2}}\left(H_{h}\right)^{2} \quad \frac{e^{2}}{4}\left(d \tilde{B}_{d-h}\right)^{2}+\frac{\rho^{2}}{4}\left(\tilde{B}_{d-h}\right)^{2}$

Higgs phase $\quad \frac{1}{\tilde{\rho}^{2}}\left(d \tilde{H}_{D-h}\right)^{2}+e^{2}\left(\tilde{H}_{D-h}\right)^{2} \quad \frac{4}{e^{2}}\left(d B_{h-1}\right)^{2}+\frac{\tilde{\rho}^{2}}{4}\left(B_{h-1}\right)^{2}$

of freedom of mass $\tilde{M}=\tilde{\rho} e$. The effective Lagrangians for each of the three pairs of dual actions are shown in the table ${ }^{3}$.

Some observations are in order here. First, only for the case $h=(D-1) / 2$ can we have Higgs/confinement duality in which case it is realized as the 4D QED case. Notice that the dual Higgs phase description is natural in the sense that it corresponds to the original field $B_{h-1}$ getting a mass as in the original Higgs effect, this analogy can be seen more explicitly by realizing this effect in terms of a Stuckelberg mechanism. This is similar to the dual confing phase in which the original dual field $\tilde{B}_{d-h}$ gets a mass. We can then conclude that the mechanism to describe the confinement and Higgs phases by taking the original field strength and promoting it to the fundamental propagating field is just the dual of the Higgs effect.

I would like to remark that the process of monopole condensation is a dynamical question that has not been addressed here. We have only assumed that this happens and then described the effective actions and duality after condensation. To know if the dynamics favours condensation we have to consider the system in more detail and analyze case by case.

\footnotetext{
${ }^{3}$ The relative signs of the terms are dimension dependent and are not shown here, see reference [16] for the explicit signs on each case.
}

The best studied examples are those in dimensions $D \leq 4$ with instantons or monopoles as the topological defects. The $2 \mathrm{D}$ case is particularly interesting since it corresponds to strings on a circle as discussed in section (2.1) for which the original and dual fields are 2D scalars $X, \tilde{X}$. The elementary and topological defects are instantons $(h-2=D-h-2=-1)$. The contribution of one type of instantons to the partition function can be computed explicitly in the dilaton gas approximation leading to an effective action (in the Higgs phase)

$S=\int d^{4} x\left(R^{2} \partial X \bar{\partial} X-A \cos \frac{X}{R}\right)$

This is just the Sine-Gordon model discussed in section (2.3). For small field values $X$ the potential reduces to a quadratic potential reproducing our result for the Higgs phase (or dual confinement if we used $\tilde{X}$ )(see table). This is a check that our presciption to build the confinement effective action is correct (up to higher derivative terms). The renormalization group flow for the couplings $R, \rho$ in the Sine-Gordon model has been well studied [1], leading to the conclusion that at $R=2$ there is a phase transition from a Coulomb phase $R>2$ which is conformal invariant $\rho=0$, to a confinement or Kosterlitz-Thouless phase at $R<2$ which is not conformally invariant and the field $X$ looses its interpretation as a coordi- 
nate in target space. The critical value $R=2$ can be found from the mass formula (4), because for $R<2$ there appear tachyonic winding modes [43. The existence of these two phases has lead some authors to conclude that instantons break the $R \leftrightarrow 1 / R$ duality. From our analysis we can see that this is not the case. First, in the confinement phase duality is still manifest but now as a duality between a massive scalar $X$ and a massive vector. Furthermore, if we consider both types of instantons the phase structure is completely changed. This can also be seen from the mass formula (4) since also for $R>1 / 2$ there are extra tachyonic states, but this time these are momentum states rather than winding. The structure of the phase diagram happens to be identical to the clock models studied by Cardy and Rabinovici since they arrived at a condition which is identical to the existence of tachyons in equation (4). The end result is that there are only two phases, Higgs and confinement (the original Coulomb phase is not realized which is expected for bosonic strings because of the tachyon), separated at the selfdual point $R=1$, both phases are described by massive scalars dual to massive vectors and so the effective actions are identical except for the exchange $R \leftrightarrow 1 / R$ showing that the phase diagram is manifestly invariant under duality! This is a realization of the Higgs/confinement duality.

This situation can be easily generalized for the 2D toroidal compactifications of section (2.1) but the phase structure is much richer, the reason being the periodicity of the real part of the field $T$ in (11) implying the existence of an infinite number of oblique confinement phases separated at the points $T=\exp \pi / 3$ and its infinite images under $S L(2, \mathbb{Z})$ transformations 45$]$.

For supersymmetric string theories there are no points where states become tachyonic and therefore only the Coulomb (conformal) phase is realized 1 . But if the coordinate $X$ is time, then there are no tachyons for temperatures smaller than the Hagedorn temperature $T_{H}$ where a tachyon appears indicating a phase transition, the tachyon

\footnotetext{
${ }^{4}$ Remember this analysis is non-perturbative in the $2 \mathrm{D}$ worldsheet but is still perturbative in the string coupling, non-perturbative string effects can change this phase structure as we are learning from $S$ duality.
}

dissapears at the dual Hagedorn temperature $\tilde{T_{H}}$. Therefore there are two Coulomb phases dual to each other with the standard string picture and for temperatures $\tilde{T_{H}}>T>T_{H}$ there is a selfdual phase described by a massive time coordinate. Again duality is manifest.

For 3D QED again the topological defects are instantons and a dilute instanton gas calculation gives an action for the dual field (a scalar in this case) of the Sine-Gordon type. It is well known that for this case the dynamics is such that only the confining phase is realized. Our analysis only adds that this phase can be described by two dual actions in terms of a massive scalar or a massive 2-index antisymmetric tensor.

For 4D QED a similar analysis can be done and the confining phase, induced by the condensation of monopoles, can be described either by massive vector or a massive antisymmetric tensor with two indices. This case has been recently studied in detail by Polyakov who found that the description in terms of a massive two-index tensor has the interpretation of a 'confining' string, since this field couples naturally to a string [47], [48]. In this way understanding confinement by monopole (4D) or instanton (3D) condensation, or by a confining string as in the old string picture of quark confinement can be seen to be one and the same physical interpretation. It has been claimed that this picture generalizes to YangMills theories 47.

Also for the D case with $h=3$, it has been seen explicitly that breaking of the global Peccei-Quinn symmetry by non-perturbative effects, does not break duality and the dual of the massive axion field is a massive 3-index field [49], in perfect agreement with our general results.

For higher dimensions and ranks of the tensors there are indications that $p$ branes could also condense and induce a phase transition. This could be relevant for uncovering all the phases of $M$ theory. However these cases need to be better understood, probably the recent studies of $D$-branes may help understanding the possibility of $p$-brane condensation. 


\subsection{Duality and Fourier transforms}

The duality between massive antisymmetric tensors can be generalized for actions with higher derivatives and general potentials. Starting with the action:

$S=\int d^{D} x\left(F\left(\partial H_{h}\right)+G\left(H_{h}\right)\right)$

This can be obtained from the partition function

$$
\int \mathcal{D} \tilde{B}_{d-h} \mathcal{D} H_{h} e^{\int d^{D} x\left(H \cdot d \tilde{B}_{d-h}+G\left(H_{h}\right)+\tilde{F}\left(\tilde{B}_{d-h}\right)\right)}(61)
$$

where $e^{\tilde{F}}$ is the Fourier transform of $e^{F}$. 50 . Performing the integral over $\tilde{B}_{d-h}$ we recover the original action above and performing the integral over $H_{h}$ we obtain the dual action

$\tilde{S}=\int d^{D} x\left(\tilde{F}\left(\tilde{B}_{d-h}\right)+\tilde{G}\left(\partial \tilde{B}_{d-h}\right)\right)$

From here we can see that a nontrivial potential $G\left(H_{h}\right)$ gives rise to a higher derivative term for the dual $\tilde{G}\left(\partial \tilde{B}_{d-h}\right)$ and viceversa. Also for a quadratic potential, we know that the Fourier transform of a gaussian is another gaussian and so we recover the result of the massive actions of the previous subsection. Furthermore, for vanishing potential we know that the Fourier transform of 1 is $\delta\left(\partial \tilde{B}_{d-h)}\right.$ and therefore we recover the duality of the Coulomb phases of section 2.2. Therefore we can see that the dualities we have been dealing with for antisymmetric tensors are only particular cases of Fourier transforms and finding the dual action reduces to finding Fourier transforms. We can then consider a nontrivial example such as the sine-Gordon model which has a nontrivial potential. In this case we know that the Fourier transform of $e^{A \cos \tilde{B}_{d-h}}$ is the modified Bessel function $I_{P}(A)$ and therefore we can just read the dual higher-derivative action changing $P$ by $\partial H_{h}$. This has been recently done for QED $(h=2)$ and the resulting (higher derivative) theory can give some information. First, since the original variable $\tilde{B}_{d-h}$ is periodic, the Fourier transform is such that the summation in the path integral has to include infinite branches and this implies that in the confining string picture we have to sum over all Riemman surfaces 477 making explicit the string theory description of the confining phase. Furthermore, the periodicity of the original variable implies the quantization of flux for the dual variable. These features can not be seen using the quadratic potential of the previous subsection where for instance periodicity is not manifest.

It is curious to see that for the $2 \mathrm{D}$ case, this implies that the Sine-Gordon model is dual to a (higher derivative) massive vector model. But we had seen in section 2.3 that the Sine-Gordon model is dual to the fermionic Thirring model, showing the richness of duality!.

Notice that being the path integrals nongaussian, most of these results are only formal and a regularization technique has to be used in order to make sense of the dual action. For the cos potential mentioned above, a detailed lattice calculation can be found in 48 .

\section{OPEN QUESTIONS}

We have learned that global symmetries are a very useful tool to prove the duality among different theories. In fact we can now take any physical system with a global symmetry and dualize it. For instance we could take the Schrodinger equation, obtained from a variational principle, and knowing that there is a phase symmetry of the wave function, we can find the dual of Schrodinger's equation (the result does not look very illuminating at first sight). However the existence of the global symmetry is only a sufficient condition for duality and it is not necessary. A more general statement can be made at least for the antisymmetric tensor field theories for which duality is only a functional Fourier transform. This may have interesting consecuences, in particular we may state an uncertainty principle regarding dual variables analogous to position/momenta in quantum mechanics.

An interesting field by definition has many open questions. I would like to mention a few.

1. Superduality: We have used the existence of global 'internal' symmetries as guidelines for duality, but we know there are also global 'spacetime' symmetries such as Poincaré and supersymmetry. In this case we may start with a globally supersymmet- 
ric theory, making it local becomes supergravity and then we have to impose the constraint that the space is flat and the gravitino field vanishes, again exchanging the order of integration implies the existence of a dual theory, 'superduality'. Work is in progress to present nontrivial examples of this new duality [51].

2. Fermionization: We have seen how to go from a purely fermionic theory to a bosonic one, but the inverse process is not under control. For that we would need to start with a bosonic theory and impose a constraint with a fermionic Lagrange multiplier, which is not clear how to implement. Probably the development of superduality may help in solving this question since the constraint of imposing vanishing gravitino field is fermionic.

3. Mirror symmetry: Mirror symmetry is one of the most relevant symmetries discovered in perturbative string vacua. It sates that a compactification on a complex manifold $\mathcal{M}$ is equivalent to a compactification in a mirror manifold $\mathcal{W}$ such that the complex and Kähler structures of both manifolds are interchanged. For Calabi-Yau spaces this corresponds to exchanging the Hodge numbers $h_{11} \leftrightarrow h_{12}$. Most of the evidence supporting mirror symmetry is 'experimental', in the sense that explicit construction of models show this symmetry [52]. More recently, the toric variety construction of Calabi-Yau manifolds has allowed to assign $\mathcal{M}$ and $\mathcal{W}$ to two dual lattices making mirror symmetry more explicit [52]. Also use of $T$-duality for different cycles of Calabi-Yau spaces has allowed to better understand mirror symmetry [53]. But an explicit proof of mirror symmetry is still lacking. Previous attempts of proving mirror symmetry from $T$-duality have failed [?]. Since CalabiYau manifolds have no isometries, the corresponding $\sigma$-model action does not have the global symmetries usually required to dualize. In fact it was claimed in the past that the problem of proving mirror symmetry was the same as proving duality without global symmetries. But this is precisely what was done in chapter 4 . However, without doing any calculation we can immediately see that the techniques of chapter 4 cannot work entirely to prove mirror symmetry because they would give for the dual of a $\sigma$-model with variables $X^{M}$ (witha Landau-Ginzburg potential), a 2D theory in terms not of coordinates $\tilde{X}^{M}$ but of 'massive' vector variables $\left(V_{\mu}\right)^{M}$, which have no straightforward identification with the coordinates of the mirror manifold. The duality beween $\mathcal{M}$ and $\mathcal{W}$ seems closer to the Higgs/confinement duality of section 4.3 (see also [55] ). On the other hand the 2D $\sigma$ model does have a global symmetry which is a $(0,2)$ supersymmetry on the worldsheet, therefore we may imagine that superduality may have interesting relation with mirror symmetry. Notice that the $U(1) R$ symmetry associated to this $N=2$ supersymmetry is also a global symmetry of the model, but it is easy to see that dualizing with respect to this symmetry gives rise to bosonization as in chapter 2 and not to mirror symmetry.

4. Seiberg's duality: Another interesting duality symmetry is the one proposed by Seiberg for $4 \mathrm{D} N=1$ supersymmetric field theories. This duality relates different phases of an $S U\left(N_{c}\right)$ theory with those of $S U\left(N_{f}-\right.$ $N_{c}$ ) where $N_{f}, N_{c}$ represent the number of flavours and colors respectively. Proposing this new duality symmetry has been one of the major advances in the nonperturbative understanding of supersymmetric field theories recently. Again, the evidence for this duality is only indirect, although it is very convincing. An explicit proof following the lines of this paper is still lacking. There are plenty of global symmetries in these systems (flavour symmetries and supersymmetry), but, similar to the case of higher dimensional bosonization, the highly nontrivial nature of 4D field theories, com- 
bined with the structure of the duality itself (see P. Argyre's lectures) makes an explicit proof of this duality a very difficult and interesting challenge.

5. Quantum Hall effect: As it was mentioned in the introduction, there are proposals for a duality symmetry in the quantum Hall effect. This duality would be with respect to the complex parameter $\tau \equiv \sigma_{x x}+i \sigma_{x y}$ where $\sigma_{x x}$ and $\sigma_{x y}$ are the longitudinal and transverse conductivities of the Hall effect. Since $\sigma_{x y}$ is quantized, we expect the shift by an imaginary integer to be a symmetry such as the axion field in string theory. The arguments in favour of an infinite dimensional discrete group $\Gamma \subset S L(2, \mathbb{Z})$ are many which have left to a proposal for a $\Gamma$ symmetric phase diagram, but the most striking fact is that, contrary to the previous dualities, there exists real experimental evidence for the existence of this duality. Recent experimental results show explicitly that the transformation $\sigma_{x x} \leftrightarrow 1 / \sigma_{x x}$ relates two different phases of this system. Again, an explicit proof of this duality is not available yet.

We have seen duality is present in many different physical systems and well defined techniques can be used to establish the equivalence among the dual theories. Duality has also been observed experimentally, even though it is in a condensed matter system, it gives us confidence that it is actually present in other systems. As we have seen during this school, duality played an important role in the solution of $N=2$ supersymmetric theories, it is currently opening the way towards understanding black holes and probably uncovering the fundamental theory of nature. Furthermore we have seen that there are many open questions regarding duality symmetries which makes the subject healthy and alive.

\section{Acknowledgements}

I would like to thank the organizers for hospitality. C.P. Burgess for comments on the manuscript and S. Theisen for encouragement.

\section{REFERENCES}

1. For a review see, R. Savit, Rev. Mod. Phys. 52 (1980) 453; J.B. Kogut, Rev. Mod. Phys. 51 (1979) 659.

2. For a review see, D. Olive, hep-th/9508089.

3. A. Luther and I. Peschel, Phys. Rev. B9 (1974) 2911; S. Coleman, Phys. Rev. D11 (1975) 2088.

4. E. Witten, Comm. Math. Phys. 92 (1984) 455.

5. See for instance, B. Julia, in : [Superspace and Supergravity', S. Hawking, M. Roček eds., Cambridge University Press (1981).

6. K. Kikkawa and Y. Yamasaki, Phys. Lett. B149 (1984) 357; N. Sakai and I. Senda, Prog. Theor. Phys. Suppl. 75 (1986) 692.

7. For reviews see A. Giveon, M. Porrati and E. Rabinovici, Phys. Rep. 244 (1994) 77; E. Alvarez, L. Alvarez-Gaumé and Y.Lozano hepth/9410237.

8. T. Buscher Phys. Lett. B194 (1987) 59; B201 (1988) 466.

9. M. Roček and E.Verlinde, Nucl. Phys. B373 (1992) 630.

10. X. de la Ossa and F. Quevedo, Nucl. Phys. B403 (1993) 377.

11. A. Shapere and F. Wilczek, Nucl. Phys. B320 (1989) 669; C.A. Lütken and G. Ross, Phys. Rev. B 45 (1992) 11837; Phys. Rev. B48 (1993) 2500; S. Kivelson, D.H. Lee and S.C. Zhang, Phys. Rev. B46 (1992) 2223.

12. Shahar et al Science 274 (1996) 589, condmat/9510113.

13. A. Font, L. Ibáñez, D. Lüst and F. Quevedo, Phys. Lett. B. (1990); C. Hull and P. Townsend, Nucl. Phys. B438 (1995) 109; E. Witten, Nucl. Phys. B443 (1995) 85.

14. For recent reviews see: J. Polchinski, hepth/9511157; J. Schwarz, hep-th/9607201; P.K. Townsend, hep-th/9612121.

15. P. Ginsparg and F. Quevedo, Nucl. Phys. B385 (1992) 527.

16. F. Quevedo and C. Trugenberger, hepth/9604196, Nucl.Phys. B (to appear).

17. K. S. Narain, Phys. Lett. B141 (1986) 123.

18. E. Witten, Phys. Rev. D44 (1991) 314.

19. R. Dijkraaf, E. Verlinde and H. Verlinde, Nucl. Phys.B371 (1992) 269; A. Giveon, Mod. 
Phys. Lett. A6 (1991) 2843.

20. J. Horne and G. Horowitz, Nucl. Phys. B368 (1992)444; J. Horne, G. Horowitz and A. Steif, Phys. Rev. Lett. 68 (1992) 568.

21. G.W. Gibbons and K. Maeda, nucl. Phys. B298 (1988) 128; D. Garfinkle, G. Horowitz and A. Strominger, Phys. Rev. D43 (1991) 3140 .

22. E. Alvarez, L. Alvarez-Gaumé, J. Barbón and Y.Lozano Nucl. Phys. B415 (1994) 71.

23. C.P. Burgess, R.C. Myers and F. Quevedo, Nucl. Phys. B442 (1995) 75; Nucl. Phys. B442 (1995) 97.

24. M. Dine, P. Huet and N. Seiberg, Nucl. Phys. B322 (1989) 301.

25. S. Cecotti, S. Ferrara and L. Girardello, Nucl. Phys. B308 (1988) 436;K. Meissner and G. Veneziano, Phys. Lett. B267 (1991) 33; A. Sen, Phys. Lett. B274 (1992) 34.

26. A. Tseytlin, Mod. Phys. Lett. A6 (1991) 1721; J. Balog, P. Forgács, Z. Horváth and L. Palla, Nucl. Phys. B 49 (1996) 16, hepth/9601091; hep-th/9606187; P. Haagensen, hep-th/9604136 (1996); C.P. Burgess and C.A. Lütken, cond-matt/9611118 (1996).

27. V.I. Ogievetski and I. Polubarinov, Sov. J. Nucl. Phys. 4 (1967) 156; M. Kalb and P. Ramond, Phys. Rev. D9 (1974) 2273.

28. A.M. Polyakov, 'Gauge Fields and Strings Harwood Academic Publishers (1987).

29. J.F.L. Barbón, Nucl. Phys. B452 (1995) 313.

30. For a review see: M. Duff, R. Khuri and J. Lu, Phys. Rep. 259 (1995) 213.

31. C.P. Burgess and F. Quevedo, Nucl. Phys. B421 (1994) 373.

32. C.P. Burgess, C.A. Lütken and F. Quevedo, Phys. Lett. B336 (1994) 18; J. Frölich, R. Götschmann and P. Marcheti, J. Phys. A28 (1995) 1165; E. Fradkin and F. Schaposnik, Phys. Lett. B338 (1994) 253; F. Schaposnik, Phys. Lett. B338 (1994) 253; J. Edelstein and C. Núñez, to appear.

33. A. Giveon and M. Roček, Nucl. Phys. B421 (1994) 173 ; K. Sfetsos, Phys. Rev. D50 (1994) 2784; T. Curtright and C. Zachos, Phys. Rev. D49 (1994) 5408; O. Alvarez and C-H. Liu, hep-th/9503226.

34. M. Gasperini, R. Ricci and G. Veneziano,
Phys. Lett. B319 (1993) 438.

35. E.Alvarez, L. Alvarez-Gaumé and Y. Lozano, Nucl. Phys. B424 (1994) 155.

36. See for instance, Y. Lozano, Mod. Phys. Lett. A11 (1996) 2893, hep-th/9610024.

37. D.Z. Freedman and P.K. Townsend, Nucl. Phys. B177 (1981) 282.

38. C. Klimčík and P.Ševera, Phys. Lett. B351 (1995) 455; Phys. Lett. B372 (1996) 65; C. Klimčíik, Nucl. Phys. Proc. Suppl. 46 (1996) 116, hep-th/9509095.

39. C. Teitelboim, Phys. Lett. 167B (1986) 63; Phys. Lett 167B (1986) 69.

40. C.P. Burgess and F. Quevedo, Phys. Lett. B329 (1993) 457.

41. A.M. Polyakov and P.B. Wiegman, Phys. Lett. 141B (1984) 223.

42. P. Orland, Nucl. Phys. B205 [FS5] (1982) 10; D. Foerster, Phys. Lett. 77B (1978) 211; S.-J. Rey, Phys. Rev. D40 (1989) 3396.

43. B. Sathiapalan, Phys. Rev. D35 (1987) 3277; I. Kogan, JETP Lett. 45 (1987) 709.

44. D.J. Gross and I. Klebanov, Nucl. Phys. B344 (1990) 475.

45. J.L. Cardy and E. Rabinovici, Nucl. Phys. B205 [FS5] (1982) 1; J.L. Cardy, Nucl. Phys. B205 [FS5] (1982) 17.

46. K.H. Obrien and C.I. Tan, Phys. Rev. D36 (1987) 1184; E. Alvarez and M. Osorio, Nucl. Phys. B304 (1988) 327; J. Atick and E. Witten, Nucl. Phys. B310 (1988) 291.

47. A. P. Polyakov, hep-th/9607049.

48. C. Diamantini, F. Quevedo and C. Trugenberger, hep-th/9612103, Phys. Lett. B396 (1997) 115.

49. C.P.Burgess, J.-P. Derendinger, F. Quevedo and M. Quirós, Phys. Lett. B348 (1995) 428.

50. H. Kawai, Progr. Theor. Phys. 65 (1981) 351.

51. C.P. Burgess et al., in progress.

52. See for instance, S.-T. Yau ed. Essays on Mirror Manifolds (Int. Press, Hong Kong, 1992).

53. A. Strominger, S.-T. Yau and E. Zaslow, Nucl.Phys. B479 (1996) 243.

54. D.R. Morrison and M.R. Plesser, hepth/9508107.

55. E. Witten, Nucl. Phys. B403 (1993) 159.

56. N. Seiberg, Nucl.Phys. B435 (1995) 129. 